\title{
Spectrum Simulation with NISTMonte
}

\author{
Nicholas W. M. Ritchie
}

National Institute of Standards and Technology, Gaithersburg, MD 20899

Simple microanalytical experiments can be understood using the analytical tools built into most EDX and WDS software packages. However when the sample differs substantially from a polished, bulk solid or when there are multiple structural components, the standard tools offer little insight. In these circumstances, a Monte Carlo simulation may be the best approach. However, many Monte Carlo simulators don't allow for the definition of complex sample geometries. NISTMonte[1] is a new Monte Carlo simulator for microanalysis that was designed to handle sample geometries of arbitrary complexity. This article reports on recent enhancements to NISTMonte to support the generation of realistic EDX-like spectra. This involves both the generation of characteristic $x$-rays and bremsstrahlung and modeling of the detector physics. This model will be applied to simulate spectra from a bulk glass and a $5 \mu \mathrm{m}$ glass particle.

The physics behind the generation of characteristic and bremsstrahlung radiation are fundamentally different. While the energy distribution of characteristic radiation depends fundamentally on the electronic structure of the target atom, bremsstrahlung is due to less energy specific interactions with the atomic nuclei. The physics of the characteristic radiation generation is to a large extent encapsulated in the ionization cross-section. NISTMonte uses an empirical expression for the ionization cross section due to Casnati et al[1]. The bremsstrahlung production is based on tabulated calculations of the bremsstrahlung cross section by Seltzer \& Berger[2]. Generation of characteristic and bremsstrahlung radiation is calculated at each electron trajectory segment. The bremsstrahlung calculation involves two steps: first, calculate the probability that a bremsstrahlung event (of any energy between the beam energy and some minimum energy, $\mathrm{E}_{\min }$ ) occurs; then second, assign the event an energy randomly selected from the distribution of bremsstrahlung intensity with respect to emission energy. Each iteration of this algorithm produces a single (fractional) bremsstrahlung event with energy between the beam energy and $\mathrm{E}_{\min }$. Bremsstrahlung events are tracked through the sample to the detector in the same way characteristic events are tracked and the emitted intensity is calculated based on the absorption path length. The intensities of both the bremsstrahlung and characteristic radiation are fully specified by the model. The only scaling necessary is a global multiplicative factor to compensate for the fact that a Monte Carlo simulation typically corresponds to at least $10^{6}$ fewer electrons than an experimental measurement.

The emitted fractional x-ray events are accumulated by a modeled EDX detector. The detector model includes a model of the window, (potentially including an ice layer), dead layer and conductive surface layer. The events are convolved with a detector response function that mimics the energy resolution of an EDX detector. The resulting spectra can be written out in EMSA format.

Because the physical models fully specify the radiation intensity, it is informative to compare the ratio of the intensity of the characteristic radiation to the intensity of bremsstrahlung radiation. If this ratio is similar to experimentally derived spectra, this is evidence suggesting that the model works. Figure 1 compares K3189 spectrum measured at $20.0 \mathrm{keV}$ with a spectrum for the same material generated by NISTMonte using the same instrument conditions. The only adjustable parameter is an overall scaling factor which was selected to give the same integrated intensity in the simulated spectrum and the measured spectrum.

Figure 2 shows series of spectra from $5 \mu \mathrm{m}$ particle of $\mathrm{K} 3189$ glass as the beam is stepped 
across the particle. On the side of the particle furthest from the detector, absorption plays a dominant role at low energies. When the beam strikes the particle on the side closest to the detector, the curve of the particle greatly reduces the absorption path length below even the bulk case.

\section{References:}

[1] N.W.M. Ritchie, submitted to Surface and Interface Analysis

[2] E. Casnati, A. Tartari \& C. Baraldi, J Phys B 15 (1982) 155 as quoted by C. Powell in Ultramicroscopy 28 (1989) 24-31

[3] Seltzer S, Berger M, Atom. Data and Nucl Data Tables, 35, 1986 pp 345-418

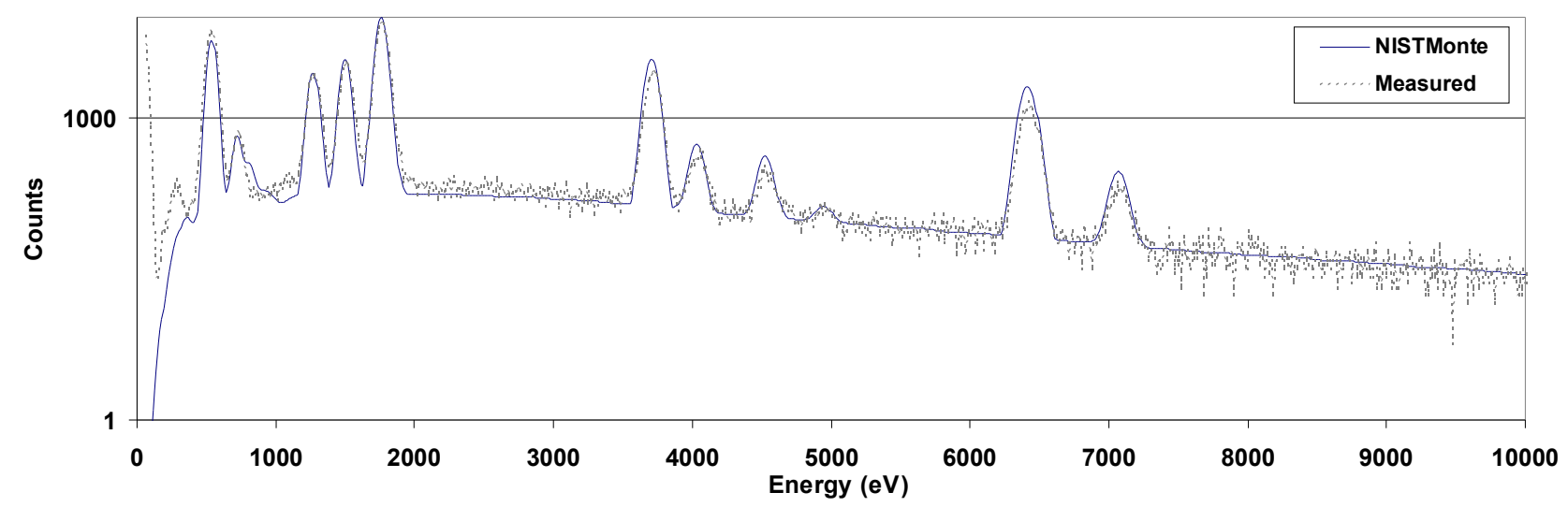

Figure 1: Compares a measured spectrum with a simulated spectrum for a bulk sample of K3189 glass at $20 \mathrm{keV}$. (Counts are plotted on a logarithmic scale)

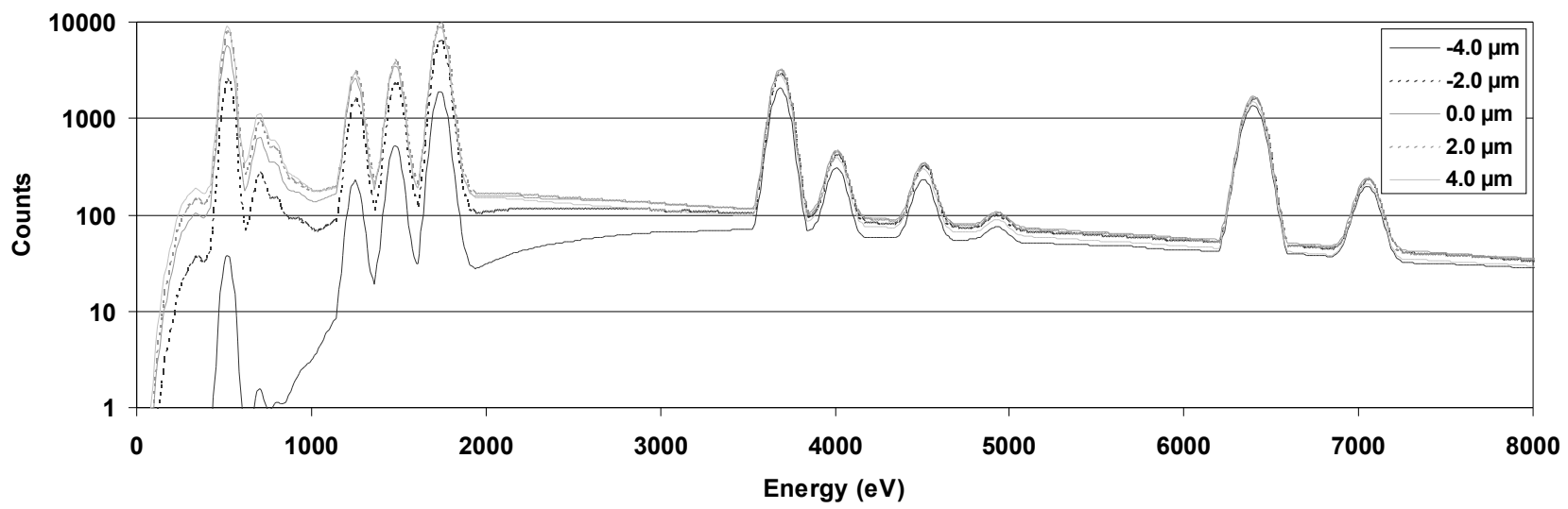

Figure 2: A series of simulated spectra generated as a $20 \mathrm{keV}$ beam is rastered across the surface of a $5 \mu \mathrm{m}$ particle of K3189 glass. (Counts are plotted on a logarithmic scale) 